\title{
Convergence Verification: From Shared Memory to Partially Synchronous Systems^
}

\author{
K. Mani Chandy, Sayan Mitra, and Concetta Pilotto \\ California Institute of Technology \\ Pasadena, CA 91125 \\ \{mani,mitras, cetta\}@caltech.edu
}

\begin{abstract}
Verification of partially synchronous distributed systems is difficult because of inherent concurrency and the potentially large state space of the channels. This paper identifies a subclass of such systems for which convergence properties can be verified based on the proof of convergence for the corresponding discrete-time shared state system. The proof technique extends to a class of partially synchronous systems in which an agent's state also evolves continuously over time. The proof technique has been formalized in the PVS interface for timed I/O automata and applied to verify convergence of a mobile agent pattern formation algorithm.
\end{abstract}

\section{Introduction}

In a partially synchronous distributed system a collection of processes interact by exchanging messages. Sent messages are either lost or delivered within a constant but unknown time bound. This model of communication presents an interesting and realistic middle-ground between the two extremes of completely synchronous (lock-step execution) and asynchronous (unbounded message delay) models. The model is particularly appropriate for a wide class of systems including those employing wireless communication and mobile agents. Algorithms and impossibility results for problems such as mutual exclusion and consensus [10] in this model have been studied extensively (see, for example, Chapters 24-25 of [16] and the bibliographic notes).

Partially synchronous systems are difficult to understand and reason about because of their inherent concurrency and message delays. Formal models, in particular variants of Timed Automata [2,13], have been used to model and analyze such systems, however, there have been few applications of formal verification techniques in checking correctness. Typically these systems present difficulty for model checking because of the huge state space which includes the (potentially large number of) messages in transit. Nevertheless, in a recent paper [11] the time to reach agreement of a consensus protocol has been model checked with UPPAAL [5] by exploiting a key compositional property of the protocol. Two other partially synchronous distributed algorithms have been model checked in [14].

* The work is funded in part by the Caltech Information Science and Technology Center and AFOSR MURI FA9550-06-1-0303. 
In this paper, we study a general class of partially synchronous distributed systems for which the state spaces of the participating processes can be continuous. Such systems arise in sensor networks, mobile robotics, and unmanned vehicle coordination applications. Techniques based on analyzing the Eigen values of state-transition matrices $[18,6]$ that have been used for verifying convergence of completely synchronous systems, cannot be applied in a straightforward way to partially synchronous systems that are highly nondeterministic. The main contributions of this paper are: (i) a methodology for transforming a shared state distributed system - in which processes can read each other's state instantaneously - to a corresponding partially synchronous system, such that the convergence properties of the original system are preserved in the latter, (ii) a substantial verification case study carried out within the Tempo/PVS framework $[4,1]$ based on the above theory.

We begin in Section 2 by describing Shared State (SS) systems - a general discrete-time model for distributed systems in which each process can change its state by reading the states of some subset of other processes. A change of state can be nondeterministic and each process is free to change its state at any point in time, independent of the others. We adapt a theorem from Tsitsiklis [20], to obtain a sufficient condition for proving convergence of such shared state systems. Given a shared state system $\mathcal{A}$, this sufficient condition requires us to find a collection of shrinking invariant sets for $\mathcal{A}$. Next, in Section 3, we present a natural transformation of the given shared state system $\mathcal{A}$ to a partially synchronous system $\mathcal{B}$. The partially synchronous system is modeled as a Timed Input/Output Automaton [13]. In Section 4, we show that if $\mathcal{A}$ converges, then under some assumptions about the structure of the invariant sets of $\mathcal{A}$ and message losses in $\mathcal{B}, \mathcal{B}$ also converges. Our proof relies critically on properties of the collection of shrinking invariants that are used in the theorem of [20].

In Section 5, we apply the above theory to verify convergence of a partially synchronous pattern formation protocol for mobile agents. First, we specify the shared state version of the protocol in PVS and verify its convergence using the pre-existing PVS metatheory [17] . We obtain the partially synchronous version of the pattern formation system; this is specified in PVS using the PVS/TIOA toolset [15] and we show that it satisfies the assumptions required for convergence.

\section{Preliminaries}

In this section we present a standard discrete-time model for shared state distributed systems and state a well-known theorem for proving convergence.

Standard notations are used for natural numbers $\mathbb{N}=\{0,1, \ldots$,$\} and the set$ of reals $\mathbb{R}$. For $N \in \mathbb{N}$, the set $\{0,1,2, \ldots, N\}$ is denoted by $[N]$. For a set $A$, $A_{\perp} \triangleq A \cup\{\perp\}$. The set of finite sequences of length $N$ (and infinite sequences) of elements in $A$ is denoted by $A^{N}$ (and resp., $A^{\omega}$ ). For $a \in A^{N}, i \in[N-1]$, the $i^{t h}$ element of $a$ is denoted by $a_{i}$. The same notation is used for infinite sequences. For any $x \in A, i \in[N], a \in A^{N+1},\left[a \mid a_{i}:=x\right]$ denotes the (unique) element $a^{\prime} \in A^{N+1}$ satisfying: for all $j \in[N]$, if $j=i$ then $a_{j}^{\prime}=x$ else $a_{j}^{\prime}=a_{j}$. 
A Labeled Transition System $\mathcal{A}$ is a quadruple $\left(S, S_{0}, A, \rightarrow\right)$ where (a) $S$ is a set of states, (b) $S_{0} \subseteq \mathcal{S}$ is a set of start states, (c) $A$ is a set of actions, and (d) $\rightarrow \subseteq S \times A \times S$ is a set of transitions. For $\left(s, a, s^{\prime}\right) \in \rightarrow$ we write $s \stackrel{a}{\rightarrow} s^{\prime}$. An execution $\alpha$ of $\mathcal{A}$ is an (finite or infinite) alternating sequence of states and actions $s_{0} a_{1} s_{1} a_{2} \ldots$, such that $s_{0} \in S_{0}$ and for all $i, s_{i} \stackrel{a_{i+1}}{\rightarrow} s_{i+1}$. An LTS is said to be action deterministic if for any $s, s^{\prime}, s^{\prime \prime} \in S, a \in A$, if $s \stackrel{a}{\rightarrow} s^{\prime}$ and $s \stackrel{a}{\rightarrow} s^{\prime \prime}$ then $s^{\prime}=s^{\prime \prime}$. Thus, each action $a \in A$ is associated with a unique state transition function $f_{a}: S \rightarrow S$, such that if $s \stackrel{a}{\rightarrow} s^{\prime}$ then $s^{\prime}=f_{a}(s)$.

Convergence. In order to define convergence of an execution to a state $s^{*} \in S$ we have to introduce some notion of "closeness" of states to $s^{*}$. One straightforward way to do this, and the approach we take in presenting this paper, is to assume that $S$ is equipped with a metric $d$. An infinite execution $\alpha$ converges to $s^{*}$ with respect to $d$, if for every $\epsilon>0$, there is a suffix of $\alpha$ such that for every state $s$ in this suffix $d\left(s, s^{*}\right) \leq \epsilon$. Convergence to a subset $S^{*} \subseteq S$ is defined by extending the definition of $d$ in the obvious way. We remark that for defining convergence to $s^{*}$ or to a subset $S^{*}$ of $S$, it is not necessary for $S$ to be a metric space, and it suffices to have a topological structure around $s^{*}$ (or $S^{*}$ ). The results presented in this paper carry over to this more general setting.

For verifying convergence, we restrict our attention to executions in which certain classes of actions occur infinitely often. This motivates the notion of fair executions. For a set of actions $A$, a fairness condition $\mathcal{F}$ is a finite collection $\left\{F_{i}\right\}_{i=1}^{n}, n \in \mathbb{N}$, where each $F_{i}$ is a nonempty subset of $A$. An infinite sequence of actions $a \in A^{\omega}$ to be $\mathcal{F}$-fair iff $\forall F \in \mathcal{F}, n \in \mathbb{N}, \exists m \in \mathbb{N}, m>$ $n$, such that $a_{m} \in F$. An infinite execution $\alpha=s_{0}, a_{0}, s_{1}, a_{1}, \ldots$ is $\mathcal{F}$-fair exactly when the corresponding sequence of actions $a_{0}, a_{1}, \ldots$ is $\mathcal{F}$-fair. Under a given fairness condition $\mathcal{F}$, an LTS $\mathcal{A}$ is said to converge to $s^{*}$ if every $\mathcal{F}$-fair execution converges to $s^{*}$.

Usually a convergence proof is carried out by showing the existence of a Lyapunov-like function that is nonnegative and decreases along all executions of the system. The following theorem from [20], translated to our setting, provides a general sufficient condition for proving convergence in terms of a collection of invariant sets (sublevel sets of a Lyapunov function).

Theorem 1. Consider an LTS $\mathcal{A}$ and a fairness condition $\mathcal{F}$ for $\mathcal{A}$. Suppose there exists a well ordered set $(T,<)$ with smallest element 0 and a collection of sets $\left\{P_{k} \subseteq S \mid k \in T\right\}$ satisfying:

C1. (Monotonicity) $\forall k, l \in T, k>l \Rightarrow P_{k} \subsetneq P_{l}$.

C2. (Granularity) $\forall \epsilon>0, \exists k \in T$, such that $\forall s \in P_{k}, d\left(s, s^{*}\right) \leq \epsilon$.

C3. (Initial) $S_{0} \subseteq P_{0}$.

C4. (Invariance) $\forall s, s^{\prime} \in S, a \in A, k \in T$ if $s \stackrel{a}{\rightarrow} s^{\prime}$ and $s \in P_{k}$ then $s^{\prime} \in P_{k}$.

C5. (Progress) $\forall k \in T$, if $P_{k} \neq\left\{s^{*}\right\}$ then $\exists F \in \mathcal{F}$, such that $\forall a \in F, \forall s \in P_{k}, s^{\prime} \in$ $S, s \stackrel{a}{\rightarrow} s^{\prime} \Rightarrow s^{\prime} \in P_{l}$, for some $l>k$.

Then all $\mathcal{F}$-fair executions of $\mathcal{A}$ converge to $s^{*}$ with respect to $d$. 
It turns out that under some weak assumptions about the stability of $\mathcal{A}$, these conditions are also necessary for convergence of $\mathcal{A}$. C1 requires that the sequence of predicates is monotonically stronger. C2 states that for every $\epsilon>0$ there exists a set $P_{k}$ that is contained in the $\epsilon$-ball around $s^{*}$. C4 requires that the $P_{k}$ 's are invariant under the transitions of $\mathcal{A}$. Finally, C5 requires that for any state $s$ in $P_{k}$ (other than $s^{*}$ ) there exists a fair set $F$ in $\mathcal{F}$, such that any action in $F$ takes the system to a state $P_{l}$, where $l>k$.

Shared State Systems. A distributed system consists of a finite collection of LTSs executing and communicating in parallel. In a shared state (distributed) system a process can read but not modify the states of other asynchronous processes. Formally, a shared state distributed system with $N+1$ processes is an action deterministic LTS $\left(S, S_{0}, A, \rightarrow\right)$ with the following additional structure:

(a) $S \triangleq X^{N+1}$, where $X$ is a set of process states. For each $s \in S, i \in[N], s_{i}$ is called the state of the $i^{\text {th }}$ process.

(b) $S_{0}=\left\{x_{0}\right\}$, where $x_{0} \in X^{N+1}$ is the vector of initial SS process states,

(c) The set of actions $A$ is partitioned into disjoint sets $\left\{A_{i}\right\}_{i \in[N]}$ such that for all $s, s^{\prime} \in S, a \in A_{i}$, if $s \stackrel{a}{\rightarrow} s^{\prime}$ then $\forall j \in[N] \backslash\{i\}, s_{j}=s_{j}^{\prime}$.

An action $a \in A_{i}$ corresponds to process $i$ reading the current states of a subset of other agents and updating its own state. For each action $a \in A_{i}$ we denote the state transition function $f_{a}$ restricted to the $i^{t h}$ component (mapping $X^{N+1}$ to $X)$ by $f_{i a}$. That is, if $s \stackrel{a}{\rightarrow} s^{\prime}$ then $s^{\prime}=\left[s \mid s_{i}=f_{i a}(s)\right]$. Function $f_{i a}$ is a function of the states of some subset of processes and is independent of the states of other processes; this is captured by the dependency function $D: A \rightarrow 2^{[N]}$ as follows: for any pair of states $s, u \in S, i \in[N]$, and any action $a \in A$, if for all $j \in D(a)$, $s_{j}=u_{j}$ then the $f_{i a}(s)=f_{i a}(u)$. That is, the post-state of action $a$ depends on the $j^{t h}$ state component of the pre-state only if $j \in D(a)$. We say that $j$ is a neighbor of $i$ exactly when there exists $a \in A_{i}$ such that $j$ is in $D(a)$.

\section{Partially Synchronous Systems}

In this section, we present the model for partially synchronous distributed systems and describe a natural translation of shared state systems to this model. In a partially synchronous distributed system a fixed set of processes communicate by sending messages over a broadcast channel. A message broadcast by process $i$ at some time $t$ is delivered to some (possibly empty) subset of processes; all (if any) deliveries are within $t+b$, where $b$ is a parameter of the broadcast channel.

Timed I/O Automata. We formally model partially synchronous distributed systems as Timed Input/Output Automata (TIOA) [13]. A Timed I/O Automaton is a non-deterministic state transition system in which the states may change either (a) instantaneously through a transition, or (b) continuously over an interval of time following a trajectory. We give the essential definitions for the TIOA framework and refer the reader to [13] for the details. A variable structure is 
used to specify the states of a TIOA. Let $V$ be a set of variables. Each variable $v \in V$ is associated with a type which defines the set of values $v$ can take. The set of valuations of $V$ is denoted by $\operatorname{val}(V)$. A trajectory for a set of variables $V$ models continuous evolution of values of the variables. Formally, a trajectory $\tau$ maps a left-closed interval of $\mathbb{R}_{\geq 0}$ with left endpoint 0 to $\operatorname{val}(V)$. The domain $\tau$ is denoted by $\tau$.dom. A trajectory is closed if $\tau$.dom $=[0, t]$ for some $t \in \mathbb{R}_{\geq 0}$, in which case we define $\tau$.ltime $\triangleq t$ and $\tau$.lstate $\triangleq \tau(t)$.

A TIOA $\mathcal{B}=\left(V, S, S_{0}, A, \mathcal{D}, \mathcal{T}\right)$ consists of (a) A set $V$ of variables. (b) A set $S \subseteq \operatorname{val}(V)$ of states. (c) A set $S_{0} \subseteq S$ of start states. (d) A set $A$ of actions partitioned into input, output and internal actions $I, O$, and $H$, (e) A set $\mathcal{D} \subseteq S \times A \times S$ of discrete transitions. An action $a \in A$ is said to be enabled at $s$ iff $\left(s, a, s^{\prime}\right) \in \mathcal{D}$. (f) A set $\mathcal{T}$ of trajectories for $V$ that is closed ${ }^{1}$ under prefix, suffix and concatenation. In addition, for every $\mathrm{s} \in S, \mathcal{A}$ must satisfy the following two nonblocking conditions: (i) $\forall a \in I, a$ is enabled at $\mathbf{s}$, and (ii) $\exists \tau \in \mathcal{T}$, such that $\tau(0)=\mathbf{s}$ and either $\tau$.dom $=[0, \infty)$ or $\tau$ is closed and $\exists a \in O \cup H$ enabled at $\tau$.ltime.

An execution fragment of $\mathcal{A}$ is a finite or infinite alternating sequence of trajectories and actions $\tau_{0} a_{1} \tau_{1} a_{2} \ldots$, such that for all $i$ in the sequence, $\tau_{i}$. Istate $\stackrel{a_{i+1}}{\longrightarrow}$ $\tau_{i+1}(0)$. We define the first state of $\alpha$, to be $\alpha$.fstate $\triangleq \tau_{0}(0)$, and for a closed $\alpha$, its last state to be $\alpha$.Istate $\triangleq \tau_{n}$. Istate, where $\tau_{n}$ is the last trajectory in $\alpha$, and $\alpha$.ltime $\triangleq \sum_{i} \tau_{i}$. Itime. An execution fragment $\alpha$ is admissible if $\alpha$.ltime $=\infty$. An execution fragment is an execution if $\tau_{0}(0) \in S_{0}$.

Given a shared state system $\mathcal{A}=\left(S, x_{0}, A, \rightarrow\right)$ for processes indexed by $[N]$ we define a natural translation of $\mathcal{A}$ to the partially synchronous setting. The partially synchronous system corresponding to a given shared state system $\mathcal{A}$ is a TIOA $\mathcal{B}$ obtained by composing a set of Process $i$ TIOAs - one for each $i \in[N]$ - and an TIOA LBCast which models the communication channels.

Generic process. First, we specify a TIOA Process ${ }_{i}$ for each participating process $i \in[N]$. The code in Figure 1 specifies this automaton using the TIOA Language [12]. The specification is parameterized by (a) an uninterpreted type $X$, (b) a element $x_{0 i}$ of $X$ representing the initial state of process $i$, (c) a collection of functions $g_{i a}: X_{\perp}^{N+1} \rightarrow X$, for $i \in[N], a \in A_{i}$ representing the actions of $i$, and (d) nonnegative real-valued parameters $l$ and $w$ dealing with timing. In order to obtain the process corresponding to $\mathcal{A}$, these parameters are instantiated as follows: (i) the type $X$ equals the process state set of $\mathcal{A}$, (ii) $x_{0 i}$ is set to the $i^{\text {th }}$ component of the start state of $\mathcal{A}$, (iii) for each $a \in A_{i}$, and for any $x: X, y:$ Array $\left[[N] \rightarrow X_{\perp}\right]$

$$
g_{i a}(x, y) \triangleq \begin{cases}f_{i a}\left(\left[y \mid y_{i}:=x\right]\right) & \text { if } \forall j \in D\left(f_{i a}\right), y[j] \neq \perp \\ x & \text { otherwise. }\end{cases}
$$

\footnotetext{
${ }^{1}$ See Sections 3-4 of [13] for formal definitions of the trajectory closure properties and the statements of the enabling conditions.
} 
Process $_{i}$ has the following state variables: (a) $x_{i}$ is a discrete variable of type $X$ and is initialized to $x_{0 i}$ (b) $y_{i}$ records state information about other processes received through messages. It is an array of type $X_{\perp}$ indexed by $[N]$ and initialized to $\perp ; y_{i}[j]$ is the last message (if any) that $i$ received from $j$

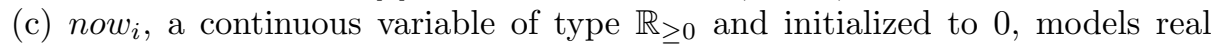
time, and (d) earliest ${ }_{i}$, a discrete variable of type $\mathbb{R}_{\geq 0}$ and initialized to $l$, is the earliest time for the next broadcast by process $i$. The initial state is defined by the initial valuations of the variables.

The transitions for send ${ }_{i}$ and receive ${ }_{i j}$ actions are specified in the preconditioneffect style in lines 14-22. (a) receive ${ }_{i j}(m)$ models the delivery of message $m$ to Process $_{i}$ from Process ${ }_{j}$ over the broadcast channel. When this action occurs, the $j^{\text {th }}$ component of the history variable $y_{i}$ is updated to $m$, and the state variable $x_{i}$ is updated according to a nondeterministically chosen function $g_{i a}$. (b) A $\operatorname{send}_{i}(m)$ action models the broadcasting of message $m$. This action can occur whenever $x_{i}=m$ and now exceeds earliest $t_{i}$. When this action does occurs, earliest $t_{i}$ is advanced to now $_{i}+l$.

Finally, the state of Process $_{i}$ changes over an interval of time according to the trajectories specified in lines 10-12. Along any trajectory, $x_{i}$ and earliest ${ }_{i}$ remain constant and now $_{i}$ increases monotonically at the same rate as real-time. The stop when condition states that no trajectory continues beyond the time point at which now $w_{i}$ equals earliest $+w$. This forces the trajectory to stop, which along with condition (ii) in the definition of TIOA forces a send to occur.

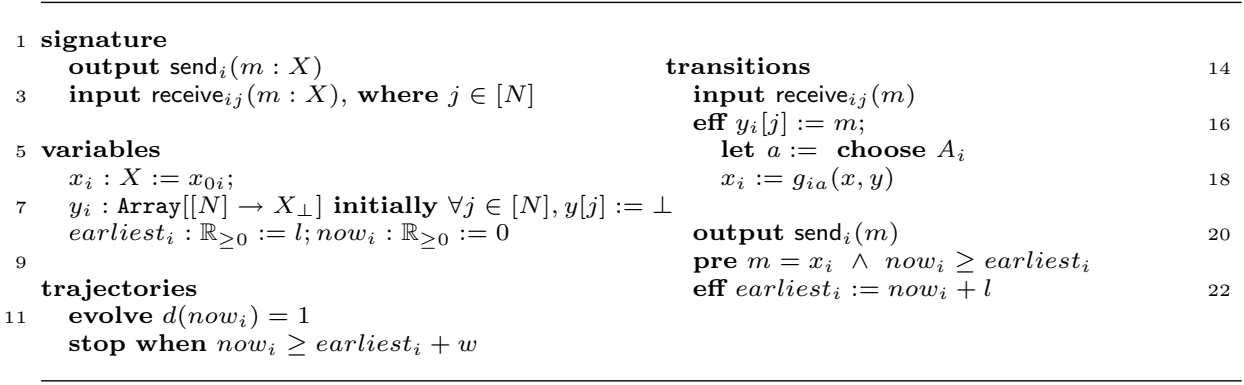

Fig. 1. Process ${ }_{i}$ TIOA with parameters $X, x_{0}, A_{i},\left\{g_{i a}\right\}_{a \in A_{i}}, l, w: \mathbb{R}_{\geq 0}$.

Channel. The LBCast automaton of Figure 2 specifies the local broadcast-based communication layer of the system. For any $b \in \mathbb{R}_{\geq 0}$, LBCast $(b)$ ensures that any message sent by Process $i$ at time $t$ is received by some subset of other processes within $[t, t+b]$.

A timed message is a pair consisting of a message of type $X$ and a deadline of type $\mathbb{R}_{\geq 0}$. For a timed message $p$, the message and the deadline are denoted by p.msg and p.dl. LBCast has two state variables: (a) buffer is a two dimensional array of sets of timed messages; it is initialized to be empty. buffer $[i, j]$ is the set of messages (time stamped with a deadline) sent by $i$ to $j$, that are in transit. (b) now is a continuous variable of type $\mathbb{R}_{\geq 0}$ and it models real time. 
The state of LBCast changes through the occurrence of send, receive, and drop actions as follows: (a) receive ${ }_{i j}(m)$ models the delivery of message $m$ sent by Process $_{j}$ to Process $i$. This action can occur when there exists $d l \in \mathbb{R}_{\geq 0}$ (actually $\geq$ now) such that the timed message $\langle m, d l\rangle$ is in buffer $[j, i]$. As a result of this action some message $m$ (with deadline $d l^{\prime} \geq$ now) is removed from buffer $[j, i]$. (b) $\operatorname{send}_{i}(m)$ models the broadcasting of message $m$ by Process ${ }_{i}$. The effect of this action is that the timed message $\langle m$, now $+b\rangle$ is added to buffer $[i, j]$ for every $j \in \mathcal{I}$. (c) $\operatorname{drop}_{i j}(m)$ models the loss of message $m$ in transit from $i$ to $j$. This action is enabled as long as the message $m$ is in transit, and the effect is that the message is removed from buffer $[i, j]$.

Along any trajectory of LBCast (see lines 25-28), buffer remains constant and now increases monotonically at the same rate as real-time. The stop when condition enforces the delivery deadline of non-dropped messages by forcing the receive actions to occur.

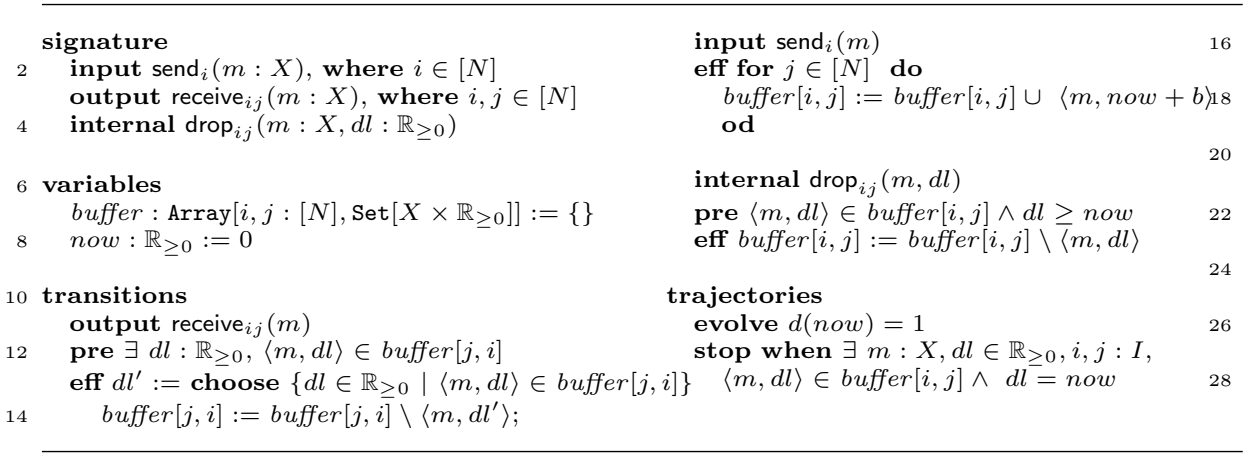

Fig. 2. LBCast $_{i, j}$ TIOA with parameter $X, b: \mathbb{R}_{\geq 0}$.

Complete system. The partially synchronous system corresponding to $\mathcal{A}$ is the composed TIOA $\mathcal{B}=\|_{i \in[N]}$ Process $_{i} \|$ LBCast. Let the set of states of $\mathcal{B}$ be $\mathcal{S}$. The values of the real-time related variables such as now $_{i}$ 's earliest ${ }_{i}$, diverge along the admissible executions of $\mathcal{B}$. In studying convergence of $\mathcal{B}$ we are really interested in the behavior of the $x_{i}$ and the $y_{i}$ variables and the messages in buffer without their time stamps. Hence, we define a projection function untime: for any state $s \in \mathcal{S}$, untime $(s)$ is an object that is identical to $s$ except that the components corresponding to now, now , earliest $_{i}$ are removed, every timed message $p$ is replaced by $p . m s g$, and all $\perp$ values are removed from the history variables $y_{i}$ 's. We denote this projected state space of $\mathcal{B}$ by $S_{\mathcal{B}}$ and its elements by $\mathbf{s}, \mathbf{u}$. Each $\mathbf{s} \in S_{\mathcal{B}}$ corresponds to a particular valuation for each non-timerelated state variable of $B$. These variable valuations are denoted by the usual (.) notation. For example, the valuations of the variables $x_{i}$ and buffer at a state $\mathbf{s}$ are denoted by $\mathbf{s} . x_{i}$ and $\mathbf{s}$.buffer. We define a metric on $S_{\mathcal{B}}$ based on the metric $d$ on $S_{\mathcal{A}}$ as follows:

$$
\begin{aligned}
U(\mathbf{s}) & \triangleq \Pi_{i=0}^{N}\left\{\left\{\mathbf{s} . x_{i}\right\} \cup_{j \in[N]}\left\{\mathbf{s} . y_{j}[i] \mid \mathbf{s} \cdot y_{j}[i] \neq \perp\right\} \cup_{j \in[N]} \mathbf{s} . \text { buffer }[i, j]\right\} \\
d_{\mathcal{B}}\left(\mathbf{s}_{1}, \mathbf{s}_{2}\right) & \triangleq \max _{r_{1} \in U\left(\mathbf{s}_{1}\right), r_{2} \in U\left(\mathbf{s}_{2}\right)} d\left(r_{1}, r_{2}\right)
\end{aligned}
$$


An admissible execution $\alpha$ is said to converge to a untimed state $\mathbf{s}^{*} \in S_{\mathcal{B}}$ if untime $(\alpha(t)) \rightarrow \mathbf{s}^{*}$ with respect to the metric $d_{\mathcal{B}}$, as $t \rightarrow \infty^{2}$. Automaton $\mathcal{B}$ converges to $\mathbf{s}^{*}$ if all its admissible executions converge.

\section{Verification of the Partially Synchronous Systems}

Throughout this section we assume that $\mathcal{A}$ is a shared state system and $\mathcal{B}$ is the corresponding partially synchronous system obtained using the translation scheme of the previous section. We denote the set of states of $\mathcal{A}$ by $S_{\mathcal{A}}$ and the individual states by $s, u$, etc. We assume that $\mathcal{A}$ converges to a state $s^{*} \in S_{\mathcal{A}}$ with respect to the metric $d$ and a fairness condition $\mathcal{F}$. We assume that convergence of $\mathcal{A}$ is proved using Theorem 1 . Therefore, we know that there exists a well ordered set $(T,<)$ with a smallest element 0 and a collection of sets $\left\{P_{k} \subseteq S \mid k \in T\right\}$ satisfying the conditions C1-5.

We define the following relation $\mathcal{R} \subseteq S_{\mathcal{B}} \times S_{\mathcal{A}}$ :

$$
\mathcal{R}(\mathbf{s}, s) \triangleq\left(\forall i \in[N], s_{i}=\mathbf{s} . x_{i} \vee \exists j \in[N], s_{i} \in \mathbf{s} . \text { buffer }[i, j] \cup \mathbf{s} . y_{j}[i]\right)
$$

For each $i$, the $i$-th component of $s$ can be one of the following: (i) the state of the $i$-th process in $\mathbf{s}$, (ii) a message in transit from $i$ to some $j$ in s.buffer, (iii) the state of the history variable $\mathbf{s} . y_{j}[i]$ for some other process $j$. If $\mathcal{R}(\mathbf{s}, s)$ then we say that $s$ is an asynchronous view of $\mathbf{s}$. Given $\mathbf{s} \in S_{\mathcal{B}}$, we define $\mathcal{R}(\mathbf{s}) \triangleq\left\{s \in S_{\mathcal{A}} \mid \mathcal{R}(\mathbf{s}, s)\right\}$. We define $\mathbf{s}^{*} \triangleq\left\{\mathbf{s} \in S_{\mathcal{B}} \mid \forall s \in \mathcal{R}(\mathbf{s}) s=s^{*}\right\}$.

In the remainder of this section we shall prove that $\mathcal{B}$ converges to $\mathbf{s}^{*}$ with respect to the metric $d_{\mathcal{B}}$. We make the following two assumptions about the structure of the $\mathcal{P}_{k}$ 's and message losses. For any specific problem these assumptions become proof obligations which must be discharged.

Assumption 1. Consider any two states $s, u \in S$, a process index $i \in[N]$, and an action $a \in A_{i}$. For any $k, l \in T, l>k$, if $P_{k}(s)$ and $P_{k}(u)$ hold, then:

B1. $P_{k}\left(\left[s \mid s_{i}:=f_{i a}(s)\right]\right) \Rightarrow P_{k}\left(\left[u \mid u_{i}:=f_{a}(s)\right]\right)$, and

B2. $P_{l}\left(\left[s \mid s_{i}:=f_{i a}(s)\right]\right) \Rightarrow P_{l}\left(\left[u \mid u_{i}:=f_{a}(s)\right]\right)$.

Assumption 2. For any $i, j \in[N]$ with $i$ a neighbor of $j$, along any admissible execution $\alpha$ of $\mathcal{B}$, for any time $t$, there exists $\zeta>l+w+b$ such that $j$ receives at least one message sent after time $t$ from $i$ within time $t+\zeta$.

All processes execute send messages within $w$ time. Hence, every every agent $i$ receives at least one message from every neighbor in the interval $[t, t+\zeta]$.

Next, we define a sequence $Q_{k}, k=0,1,2, \ldots$ of predicates on states of $S_{\mathcal{B}}$ based on the predicates $P_{k}$ on $S_{\mathcal{A}}$. Informally, $Q_{k}$ holds for a state $\mathbf{s}$ exactly when all asynchronous view of $\mathbf{s}$ satisfy $P_{k}$.

$$
Q_{k}(\mathbf{s}) \triangleq\left(\forall s \in S_{\mathcal{A}}, \mathcal{R}(\mathbf{s}, s) \Rightarrow P_{k}(s)\right) .
$$

${ }^{2} \alpha(t) \triangleq \alpha^{\prime}$.Istate, where $\alpha^{\prime}$ is the longest prefix of $\alpha$ with $\alpha^{\prime}$.ltime $=t$. 
We now show that the conditions C1-5 are satisfied by the collection of sets $Q_{k}$. The proof for the next lemma uses C1-3 property of $\left\{P_{k}\right\}$ and appears in the Appendix.

Lemma 1. The collection $\left\{Q_{k}\right\}$ satisfies C1-3.

Lemma 2. $\forall k \in T, \mathbf{s}, \mathbf{s}^{\prime} \in S_{\mathcal{B}}, a \in A_{\mathcal{B}}$, if $\mathbf{s} \stackrel{a}{\rightarrow} \mathbf{s}^{\prime}$ and $Q_{k}(\mathbf{s})$ then $Q_{k}\left(\mathbf{s}^{\prime}\right)$.

Proof. Assuming $Q_{k}(\mathbf{s})$ holds for some $k \in T$, we show that $Q_{k}\left(\mathbf{s}^{\prime}\right)$ also holds. The proof is straightforward for $a=\mathrm{drop}, a=$ send, and for a closed trajectory of $\mathcal{B}$. Consider the case where $a=$ receive $_{i j}(m), i, j \in[N]$ and $m \in X$. In order to show that $Q_{k}\left(\mathbf{s}^{\prime}\right)$, we consider any $u \in S_{\mathcal{A}}$ and assume that $\mathcal{R}\left(\mathbf{s}^{\prime}, u\right)$ holds. Then, it suffices to deduce $P_{k}(u)$.

Let the state of process $i$ in the pre-state $\mathbf{s} \in S_{\mathcal{B}}$ be $(x, y)$. Then its poststate is $\left(x^{\prime}, y\right)$, where $x^{\prime}=f_{i a}\left(\left[y \mid y_{i}:=x\right]\right)$. We define the corresponding pre-state $s \in S_{\mathcal{A}}$ as $\left[y \mid y_{i}:=x\right]$.From the definition of $\mathcal{R}$, it is follows that $\mathcal{R}(\mathbf{s}, s)$ holds. From the definition of $Q$ and $\mathrm{C} 4$ we have these two implications:

$$
Q_{k}(\mathbf{s}) \wedge \mathcal{R}(\mathbf{s}, s) \Rightarrow P_{k}(s) \quad P_{k}(s) \Rightarrow P_{k}\left(\left[s \mid s_{i}:=f_{i a}(s)\right]\right)
$$

Assume that $u$ is an asynchronous view of $\mathbf{s}^{\prime}$. Then $u$ is an asynchronous view of $\mathbf{s}$ with $u_{i}$ either unchanged, or replaced by $f_{i a}(s)$. Hence:

$$
\begin{aligned}
\mathcal{R}\left(\mathbf{s}^{\prime}, u\right) & \Rightarrow \mathcal{R}(\mathbf{s}, u) \vee\left(\exists v: \mathcal{R}(\mathbf{s}, v) \wedge\left(u=\left[v \mid v_{i}:=f_{i a}(s)\right]\right)\right) \\
Q_{k}(\mathbf{s}) \wedge \mathcal{R}\left(\mathbf{s}^{\prime}, u\right) & \Rightarrow\left(Q_{k}(\mathbf{s}) \wedge \mathcal{R}(\mathbf{s}, u)\right) \vee\left(\exists v: Q_{k}(\mathbf{s}) \wedge \mathcal{R}(\mathbf{s}, v) \wedge\left(u=\left[v \mid v_{i}:=f_{i a}(s)\right]\right)\right) \\
& \Rightarrow P_{k}(u) \vee\left(\exists v: P_{k}(v) \wedge\left(u=\left[v \mid v_{i}:=f_{i a}(s)\right]\right)\right) \quad[\text { From } Q \text { definition] } \\
& \Rightarrow P_{k}(u) \quad\left[\text { from } B 1, \text { and } P_{k}(s)\right]
\end{aligned}
$$

Lemma 3. For all $k \in T$, if $P_{k} \neq\left\{s^{*}\right\}$ and $\mathbf{s} \in Q_{k}$ then there exists $l>k$ and a closed execution fragment $\alpha$ of $\mathcal{B}$ such that

$$
(\text { untime }(\alpha . \text { fstate })=\mathbf{s}) \wedge\left(\text { untime }(\alpha \text {.Istate }) \in Q_{l}\right) \wedge(\alpha \text {.ltime } \leq 2 \cdot \zeta)
$$

Proof. Let us fix $k \in T$. By C5, there exists $l \in T, l>k$ and an action $f_{i a}$ in the shared state system such that for all $s \in \mathcal{A} \quad P_{k}(s) \Rightarrow s^{\prime}=\left[s \mid s_{i}:=f_{i a}(s)\right] \in P_{l}$. We define a new relation $R^{\prime} \cup S_{\mathcal{B}} \times S_{\mathcal{A}}$ as follows:

$$
\mathcal{R}^{\prime}(\mathbf{s}, s) \triangleq \exists u, v \in S_{\mathcal{A}}: \mathcal{R}(\mathbf{s}, u) \wedge \mathcal{R}(\mathbf{s}, v) \wedge s=\left[v \mid v_{i}:=f_{i a}(u)\right]
$$

Thus $\mathcal{R}^{\prime}(\mathbf{s}, s)$ holds exactly when $s$ is an asynchronous view $v$ of $\mathbf{s}$ except that the $i$-th agent's state is $f_{i a}(u)$ where $u$ is itself an asynchronous view of $\mathbf{s}$. We define $Q_{k}^{\prime}$ as

$$
Q_{k}^{\prime}(\mathbf{s}) \triangleq\left(\forall u \in S_{\mathcal{A}}: \mathcal{R}^{\prime}(\mathbf{s}, u) \Rightarrow P_{l}(u)\right)
$$

If $\mathbf{s} \in Q_{k} \cap Q_{k}^{\prime}$, then for all $i, \mathbf{s} . x_{i}$ satisfies $P_{l}$ and any asynchronous view of $\mathbf{s}$ satisfies $P_{k}$.

Claim. $Q_{k} \cap Q_{k}^{\prime}$ is invariant under the transitions and trajectories of $\mathcal{B}$. 
Proof of Claim. The proof is straightforward for an actions drop, send and trajectories of $\mathcal{B}$. Consider an action $a=$ receive $_{j, k}(m)$. We consider two cases $i=j$ and $i \neq j$. Consider the case when $i=j$. All $\mathbf{s} . x$ satisfies $P_{l}$. From C4, $P_{l}$ is invariant under transitions of $\mathcal{A}$. Hence all $\mathbf{s}^{\prime} . x$ satisfy $P_{k+1}$. Therefore $\mathbf{s}^{\prime} \in Q_{k}^{\prime}$. Consider the case $j \neq i$. Applying lemma 2, $Q_{k}\left(\mathbf{s}^{\prime}\right)$ holds. Hence, for all $s^{\prime} \in \mathcal{R}\left(s^{\prime}\right)$ we have that $P_{k}\left(s^{\prime}\right)$ holds. By B2, $\mathbf{s}^{\prime} \in Q_{k}^{\prime}$.

We define $\alpha$ as the concatenation of two fragments $\alpha_{1}$ and $\alpha_{2}$. We show that

1. $\exists$ a closed execution fragment $\alpha_{1}$ with untime $\left(\alpha_{1}\right.$.fstate $) \in Q_{k}$ and $\alpha_{1}$.ltime $\geq$ $\zeta$ is such that untime $\left(\alpha_{1}\right.$. Istate $) \in Q_{k} \cap Q_{k}^{\prime}$.

2. $\forall$ closed execution fragments $\alpha_{2}$ with untime $\left(\alpha_{2}\right.$.fstate $) \in Q_{k}^{\prime} \cap Q_{k}$ and $\alpha_{2}$. Itime $\geq$ $\zeta$, untime $\left(\alpha_{2}\right.$. Istate $) \in Q_{l}$.

Part 1. By Assumption 2, $i$ receives at least one message from all its neighbors by time $t+\zeta$. Denote by $\mathbf{s}^{\prime}=$ untime $\left(\alpha_{1}\right.$.lstate $)$ and assume that $\mathbf{s}^{\prime}$ is obtained by executing $g_{i a}$. By lemma $2, \mathbf{s}^{\prime} \in Q_{k}$. Denote by $s^{\prime} \in S_{\mathcal{A}}$ any state of $\mathcal{A}$ such that $\mathcal{R}^{\prime}\left(\mathbf{s}^{\prime}, s^{\prime}\right)$. We will show $P_{l}\left(s^{\prime}\right)$. By definition of $\mathcal{R}^{\prime}$, there exists $u, v$ such that $\mathcal{R}\left(\mathbf{s}^{\prime}, u\right) \wedge \mathcal{R}\left(\mathbf{s}^{\prime}, v\right) \wedge s^{\prime}=\left[v \mid v_{i}:=f_{i a}(u)\right]$. Hence, since $Q_{k}\left(\mathbf{s}^{\prime}\right)$ holds, it follows that $u \in P_{k}$ and $v \in P_{k}$. By C5, $P_{k}(u) \Rightarrow P_{l}\left(\left[u \mid u_{i}:=f_{i a}(u)\right]\right)$. Hence, by $\mathrm{B} 2, s^{\prime} \in P_{l}$ with $\left.s^{\prime}=\left[v \mid v_{i}:=f_{i a}(u)\right]\right)$.

Part 2. Fix any closed execution fragment $\alpha_{2}$ with starting state untime $\left(\alpha_{2}\right.$.fstate $) \in$ $Q_{k}^{\prime} \cap Q_{k}$. Assume that $\alpha_{2}$ ends at time $\alpha_{2}$. Itime $\geq \zeta$. Denote by $\mathbf{s}^{\prime}=$ untime $\left(\alpha_{2}\right.$. Istate). We will show that $Q_{k+1}\left(\mathbf{s}^{\prime}\right)$ holds. By Claim $1, Q_{k}^{\prime}\left(\mathbf{s}^{\prime}\right)$ holds. Let $s^{\prime}$ be any state in $\mathcal{R}\left(\mathbf{s}^{\prime}\right)$. We will show that $P_{k+1}\left(s^{\prime}\right)$ holds. By Assumption 2 (noting that $\zeta \geq b$ ), for all $j, k \quad \mathbf{s}^{\prime} . x, \mathbf{s}^{\prime} \cdot y_{j}[k]$, and $\mathbf{s}^{\prime}$. buffer $[j, k]$ contain information sent at or after time 0 and this information satisfies $P_{l}$. This is because starting from time 0 the $x$ variables satisfy $P_{l}$ and by time $\zeta$ the old messages and local copies are updated with values that satisfy $P_{l}$. Hence, any asynchronous view of $\mathbf{s}^{\prime}$ satisfies $P_{l}$. Hence, $P_{l}\left(s^{\prime}\right)$ holds.

Theorem 2. If $\mathcal{A}$ converges to $s^{*}$ with respect to $d$, then under Assumptions B1-2 and $2, \mathcal{B}$ converges to $\mathbf{s}^{*}$.

Proof. It is straightforward to see that $\mathcal{B}$ is indeed a labeled transition system with set of states $\mathcal{S}$, start states defined by the start states of $\mathcal{A}$, set of actions $A_{\mathcal{B}} \cup \mathcal{T}_{\mathcal{B}}$, and transitions $\left(s, a, s^{\prime}\right) \in \rightarrow$ if and only if (i) $\left(s, a, s^{\prime}\right) \in \mathcal{D}$ or (ii) $\exists \tau \in \mathcal{T}_{\mathcal{B}}$, with $\tau(0)=s$ and $\tau$.Istate $=s^{\prime}$. Therefore, Theorem 1's sufficient conditions for convergence are applicable to $\mathcal{B}$ with fairness conditions replaced by time bounded progress guarantees. From Assumptions B1-2 and convergence of $\mathcal{A}$ we obtain a collection $\left\{Q_{k}\right\}$ of invariant sets of $\mathcal{B}$ which satisfy conditions 14. Assumption 2 and Lemma 3 imply that $\mathcal{B}$ makes progress with respect to these invariant sets. 


\section{Verifying Convergence of a Pattern Formation Protocol}

We verify a class of pattern formation protocols for mobile agents. Starting from arbitrary locations in a space, the goal of such a protocol is to make the agents converge to some predefined spatial pattern. Distributed pattern formation protocols have been studied extensively, but typically under the assumption that the agents can communicate synchronously (see, for example $[9,6,8,18]$ ). In this paper, we present the verification of a simple one-dimensional algorithm. Several generalizations of this protocol have been presented in [7].

The shared state version of the protocol is modeled as a LTS $\mathcal{A}=\left(S, S_{0}, A, \rightarrow\right)$, where (a) $S=\mathbb{R}^{N+1}$, (b) $S_{0} \in \mathbb{R}^{N+1}$ (c) $A=\cup_{i \in[N]} A_{i}$, where $A_{i} \subseteq\left\{\left(i, \operatorname{avg}_{l, r}\right) \mid l<\right.$ $i<r\}$. (d) $f_{i} \operatorname{avg}_{l, r}: \mathbb{R}^{N+1} \rightarrow \mathbb{R}$ such that for $s \in S, f_{i} \operatorname{avg}_{l, r}(s)=\frac{r-i}{r-l} s_{l}+\frac{i-l}{r-l} s_{r}$. Note that for every $l<i$ and $r>i$, the object $\left(i, \operatorname{avg}_{l, r}\right)$ may not be an action for agent $i ; A_{i}$ is some subset of such actions. Action $\left(i, \operatorname{avg}_{l, r}\right) \in A_{i}$ changes the state of the $i^{t h}$ agent according to the function $f_{i, \text { avg }_{l . r}}$. This function depends on the states of agents $l$ and $r$, that is $D\left(\left(i, \operatorname{avg}_{l, r}\right)\right)=\{l, r\}$. We adopt the notations from Section 2 to $\mathcal{A}$. For instance, for a state $s \in S$, we denote the $i^{t h}$ component as $s_{i}$. It is easy to check that $\mathcal{A}$ is a shared state system. At a particular state $s$ of $\mathcal{A}$, we say that agent $i$ is located at $s_{i}$. Throughout this section, mid denotes the value $\frac{N}{2}$.

We define a state $s^{*} \in S$ as follows: $\forall i \in[N], \quad s_{i}^{*} \triangleq s_{00} \frac{N-i}{N}+s_{0 N} \frac{i}{N}$. This specifies a particular pattern where agents are located, in order, at equidistant points on a straight with extremes $s_{00}$ and $s_{0 N}$. We set $\mathcal{F}=\left\{A_{i}\right\}_{i \in[N]}$. It turns

out that $\mathcal{F}$-fair executions of $\mathcal{A}$ converges to the state $s^{*}$ with respect to the Euclidean metric on $S$. In the remainder of this section, we shall first verify this property and show how this result carries over to the convergence of the partially synchronous version of $\mathcal{A}$.

\subsection{Convergence of Shared State Protocol}

First, we introduce the deviation profile of a state of $\mathcal{A}$ which in turn will be used to define a sequence of predicates which satisfy C1-5. For any $x \in \mathbb{R}$ and $i \in[N]$, we define $e_{i}(x) \triangleq\left|x-s_{i}^{*}\right|$. Given $s \in S_{\mathcal{A}}, i \in[N], m \in \mathbb{N}$, we define the following two symmetric predicates:

$$
\begin{aligned}
& L_{m, j}(s) \triangleq \forall l \leq j \quad e_{l}\left(s_{l}\right) \leq C \cdot \beta^{m}\left(1-\frac{1}{2^{l}}\right) \\
& R_{m, j}(s) \triangleq \forall r \geq N-j \quad e_{r}\left(s_{r}\right) \leq C \cdot \beta^{m}\left(1-\frac{1}{2^{N-r}}\right)
\end{aligned}
$$

where $\beta \triangleq\left(1-\frac{1}{2^{N}}\right)$, and $C$ is chosen such that the $L_{0, \frac{N}{2}}$ and $R_{0, \frac{N}{2}}$ predicates are satisfied at the start state $s_{0}$. For any state $s$, if $L_{m, j}(s)$ holds then the deviations of the agent locations at $s$ from those at $s^{*}$ is upper-bounded by the deviation profile function (shown in Figure 3) increasing from 0 to $j$. Symmetrically, the predicate $R_{m, j}(s)$ holds if the deviations are decreasing from $N-j$ to $N$. 

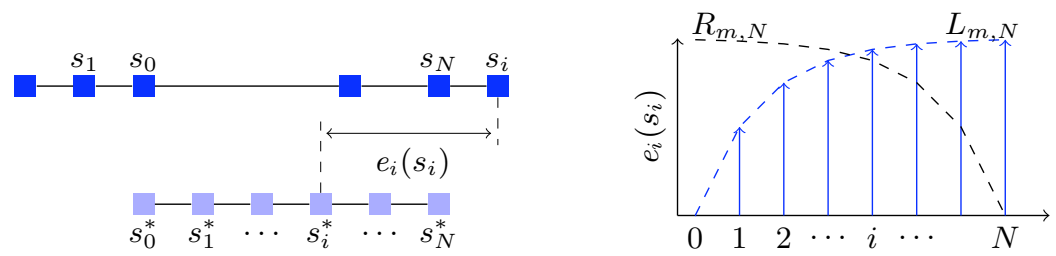

Fig. 3. Deviation from $s^{*}$. Left and Right deviation profiles.

For a state $s \in S_{\mathcal{A}}$, we define $\max (s) \triangleq \max _{i \in[N]} e_{i}\left(s_{i}\right)$ and $M_{m}(s) \triangleq$ $\left\langle\max (s) \leq C \cdot \beta^{m}\right\rangle$. We define $d\left(s, s^{*}\right) \triangleq \max (s)$. For any $j \in[$ mid] , we define the following symmetric predicates:

$\mathcal{L}_{m, j}(s) \triangleq M_{m}(s) \wedge L_{m, j}(s) \wedge L_{m-1, m i d}(s), \quad \mathcal{R}_{m, j}(s) \triangleq M_{m}(s) \wedge R_{m, j}(s) \wedge R_{m-1, \text { mid }}(s)$.

These predicates partition $[N]$ into three groups as: for all $i \in[j]$, the deviation for agent $i$ is upper bounded by the profile function defined by $C \cdot \beta^{m}$; for $i \in\{j+1 \ldots$ mid $\}$ the upper bound is $C \cdot \beta^{m-1}$; and for the remaining, the deviations do not have any upper bound other than one given by the first part of the predicate $\left(\leq C \cdot \beta^{m}\right)$. For $i \in[N]$, we define the left profile function $l p_{m, j}(i)$ as $C \cdot \beta^{m}\left(1-\frac{1}{2^{i}}\right)$ if $i \leq j$, equals to $C \cdot \beta^{m-1}\left(1-\frac{1}{2^{i}}\right)$ if $j<i \leq$ mid and $C \cdot \beta^{m}$ otherwise. This function is concave.

Lemma 4. Let $T$ be the set $\mathbb{N} \times[$ mid $]$ equipped with lexicographic ordering $\left(\leq_{\text {lex }}\right)$. The collections $\left\{\mathcal{L}_{m, j}\right\}$ and $\left\{\mathcal{R}_{m, j}\right\}$ indexed by $T$ satisfy $C 1-4$.

Proof. C1. Consider any state $s \in \mathcal{L}_{m_{2}, j_{2}}$ and any pair $\left[m_{1}, j_{1}\right] \leq_{\text {lex }}\left[m_{2}, j_{2}\right]$. If $m=m_{1}=m_{2}, s \in \mathcal{L}_{m, j_{2}} \Rightarrow s \in \mathcal{L}_{m, j_{1}}$ since the profile holds up to $j_{2}$, it is valid up to $j_{1}$ (for all $j_{1} \leq j_{2}$ ). When $m_{1}<m_{2}, s \in \mathcal{L}_{m_{2}, j_{2}} \Rightarrow s \in \mathcal{L}_{m_{1}, j_{1}}$ for all $j_{1}, j_{2} \leq \mathrm{mid}$; this is because for all $i l p_{m_{2}, j_{2}}(i) \leq l p_{m_{1}, j_{1}}(i)$ since $\beta^{m_{2}}<\beta^{m_{2}-1} \leq$ $\beta^{m_{1}}$. For $\mathrm{C} 2$, for all $\epsilon$ we set $k$ to be any value satisfying $C \cdot \beta^{k} \leq \epsilon$. Hence, $\forall s$ satisfying $\mathcal{L}_{k, 0}$ we have that $\max (s) \leq C \cdot \beta^{k}<\epsilon$. C3 follows from the definition of $C$. C4. Assume without loss of generality $s \in \mathcal{L}_{m, j}$ and $a=\left(i, \operatorname{avg}_{l, r}\right)$. For all $j \neq i, s_{j}^{\prime}$ satisfies $\mathcal{L}_{m, j}$, since $s_{j}^{\prime}=s_{j}$. Assume $i \leq j$. The value $s_{i}^{\prime}$ satisfies $\mathcal{L}_{m, j}$ as well, and $e_{i}\left(s_{i}^{\prime}\right)$ is upper bounded by

$\frac{r-i}{r-l} e_{l}\left(s_{l}\right)+\frac{i-l}{r-l} e_{r}\left(s_{r}\right) \leq \frac{r-i}{r-l}\left(1-\frac{1}{2^{l}}\right) C \cdot \beta^{m}+\frac{i-l}{r-l} C \cdot \beta^{m} \leq C \cdot \beta^{m}\left(1-\frac{1}{2^{i}}\right)$

An analogous argument is used to prove the case when $i>j$.

Condition C5 is only partially satisfied by these predicates; for any $m$ and $j<$ mid, for all $\mathcal{L}_{m, j}$ (resp. $\mathcal{R}_{m, j}$ ) there exists an action such that the execution of this action take the system to $\mathcal{L}_{m, j+1}$ (resp. $\mathcal{R}_{m, j+1}$ ). The following relationships among $\mathcal{L}$ and $\mathcal{R}$ are used for showing $\mathrm{C} 5$. The proofs appears in the Appendix.

Lemma 5. $\forall m \in \mathbb{N}, \quad \mathcal{L}_{m, m i d} \cap \mathcal{R}_{m, m i d}=\mathcal{L}_{m+1,0} \cap \mathcal{R}_{m+1,0}$ 
Lemma 6. $\forall j<$ mid (a) $\exists a_{1}$ such that $\forall s \stackrel{a_{1}}{\rightarrow} s^{\prime}$ and $\forall m \in \mathbb{N}, s \in \mathcal{L}_{m, j} \Rightarrow s^{\prime} \in$ $\mathcal{L}_{m, j+1}$. (b) $\exists a_{2}$ such that $\forall s \stackrel{a_{2}}{\rightarrow} s^{\prime}$ and $\forall m \in \mathbb{N}, s \in \mathcal{R}_{m, j} \Rightarrow s^{\prime} \in \mathcal{R}_{m, j+1}$.

Lemma 5 implies that the left and right profile predicates satisfy C1-4, but in order to prove $\mathrm{C} 5$ we require both these predicates hold simultaneously. This motivates our next definition. For state $s \in S_{\mathcal{A}}, m \in \mathbb{N}, j \in[$ mid -1$], b \in\{0,1\}$, we define: $\mathcal{P}_{m, j, b}(s) \triangleq \mathcal{L}_{m, j+b}(s) \wedge \mathcal{R}_{m, j}(s)$. All indices from 0 to $j+b$ and from $N-j$ to $N$ belong to the profile defined by $C \cdot \beta^{m}$, while the indices between $(j+b)+1$ and $(N-j)-1$ belong to profile defined by $C \cdot \beta^{m-1}$.

Lemma 7. Let $T$ be the set $\mathbb{N} \times[$ mid -1$] \times\{0,1\}$ equipped with lexicographic ordering $\left(\leq_{\text {lex }}\right)$. The collection $\left\{\mathcal{P}_{m, j, b}\right\}$ indexed by $T$ satisfies $C 1-5$.

Proof. It is straightforward to check using Lemma 4 that the sequence of predicates satisfy C1-4. C5. Applying Part (a) of Lemma 6,

$$
s \in \mathcal{P}_{m, j, 0} \Rightarrow s \in \mathcal{L}_{m, j} \wedge s \in \mathcal{R}_{m, j} \Rightarrow s^{\prime} \in \mathcal{L}_{m, j+1} \wedge s^{\prime} \in \mathcal{R}_{m, j} \Leftrightarrow s^{\prime} \in \mathcal{P}_{m, j, 1} .
$$

for any $m, j$ with $j \leq$ mid -1 , let $a_{1}$ be any action in $A_{j+1}$. Without loss of generality, we assume $a_{1}=\left(j+1, \operatorname{avg}_{l, r}\right)$. Using part $(b)$ of Lemma 6 , we obtain

$s \in \mathcal{P}_{m, j, 1} \Rightarrow s \in \mathcal{L}_{m, j+1} \wedge s \in \mathcal{R}_{m, j} \Rightarrow s^{\prime} \in \mathcal{L}_{m, j+1} \wedge s^{\prime} \in \mathcal{R}_{m, j+1} \Leftrightarrow s^{\prime} \in \mathcal{P}_{m, j+1,0}$.

Next, for any $m, j$ with $j<m i d-1$, let $a_{2}$ be any action in $A_{N-(j+1)}$. Again, without loss of generality, let $a_{2}=\left(N-(j+1), \operatorname{avg}_{l, r}\right)$. Finally from Lemma 5 , $s \in \mathcal{P}_{m, m i d-1,1} \Rightarrow s \in \mathcal{P}_{m+1,0,0}$. Since both $A_{j+1}$ and $A_{N-(j+1)}$ are in the fairness condition $\mathcal{F}$, we obtain the required result.

Lemma 7 and Theorem 1 imply that all $\mathcal{F}$-fair executions of $\mathcal{A}$ converge to $s^{*}$.

\subsection{Convergence of the Partially Synchronous Protocol}

From the shared state protocol for patten formation described in Section 5.1, we first obtain the corresponding Process $i$ automaton based on the translation scheme of Section 3. In particular, Process ${ }_{i}$ is a TIOA specified by the code in Figure 1 with $X=\mathbb{R}, x_{0}=s_{0 i}$ and $g_{i \text { avg }}: \mathbb{R}^{3} \rightarrow \mathbb{R}$. The $g_{i}$ avg $g_{l, r}$ functions are obtained from the $f_{i}$ avg $_{l, r}$ functions using the transformation of Equation 1. The communication channel for the system is modeled by LBCast of Figure 2 with $X=\mathbb{R}$ and some value for $b$. The complete partially synchronous system specification is the TIOA obtained by composing Process ${ }_{i}$ 's with LBCast. Finally, the convergence state $\mathbf{s}^{*}$ and $d_{\mathcal{B}}$ for $\mathcal{B}$ are obtained from $s^{*}, d$ of $\mathcal{A}$ using the definitions in 3 . It is easily checked that the collection of predicates $\left\{\mathcal{P}_{m, j, b}\right\}$ satisfy Assumptions 1 and 2. Therefore, from Theorem 2, we conclude that $\mathcal{B}$ converges to $\mathbf{s}^{*}$. In fact, we observe that the system $\mathcal{B}$ converges under the following weaker assumption about message losses:

Assumption 3. For any agent $i$, for any time $t$ there exists $\zeta>0$, such that $i$ receives at least one message sent after time $t$ from some agent $l<i(r>i$, respectively) within time $t+u$ with $\left(i, \operatorname{avg}_{l, r}\right) \in A_{i}$. 
This is weaker than Assumption 2 since each process $i$ receives at least one message from some pair (neighbor) and not necessarily all pairs in $A_{i}$. The progress property is still guaranteed because by the system makes progress executing any action of $A_{i}$.

\subsection{Verification in PVS Theorem Prover}

We have developed a PVS [19] theory for verifying partially synchronous pattern formation protocols within the exiting Timed I/O Automata/PVS framework $[3,15]$. The theory formalizes partially synchronous systems as described in this paper, and we have verified the convergence of the example presented here. The PVS theory files and the related documentation are available from http://www.infospheres.caltech.edu/papers. The the proofs presented in this section have been mechanically checked using the PVS theorem prover. The invariance of the $\mathcal{P}$ predicates are proved using the standard inductive proof technique followed by a case analysis on the actions (and trajectories) of the automaton in question. We also prove the convergence of the partially synchronous system directly under Assumption 3. An appropriately changed version of Lemma 5 holds in the partially synchronous settings as well. In order to do so, we prove a set of basic lemmas about LBCast that are used repeatedly. One example, of such a basic lemma is that if all the input messages satisfy a certain predicate, then within bounded time the values stored in the buffer satisfy the same predicate

\section{Discussion}

Designing and verifying partially synchronous distributed algorithms is complicated because of their inherent concurrency and message delays. We have presented a methodology for transforming a shared state distributed systemin which processes can read each other's state without delay - to a partially synchronous system, such that the convergence of the former carry over to the latter, under certain assumptions. Checking Assumption 1 is easy when it can be expressed as a conjunction of predicates on individual process states. It would be interesting to explore relaxations of this assumption. Assumption 2 is fairly weak, however, it is possible to weaken it further for specific protocols - as it is observed in the presented case study. We implemented the theory in PVS and have applied this methodology to verify the convergence of a mobile-agent pattern pattern formation protocol operating on partially synchronous communication. Several generalizations of the translation scheme and the convergence theorem are possible; some more immediate than others. The processes participating in the partially synchronous system could have clocks with bounded drift. We could also define arbitrary continuous trajectories for the main state variables $x_{i}$ as long as Assumption 1 is satisfied.

\section{References}

1. Tempo toolset, version 0.2 .2 beta, January 2008. http://www.veromodo.com/ tempo/. 
2. R. Alur and D. L. Dill. A theory of timed automata. Theoretical Computer Science, 126:183-235, 1994.

3. M. Archer, C. Heitmeyer, and S. Sims. TAME: A PVS interface to simplify proofs for automata models. In Proceedings of UITP '98, July 1998.

4. M. Archer, H. Lim, N. Lynch, S. Mitra, and S. Umeno. Specifying and proving properties of timed I/O automata using Tempo. Design Automation for Embedded Systems, 2008. To appear.

5. J. Bengtsson, K. G. Larsen, F. Larsson, P. Pettersson, and W. Yi. UPPAAL in 1995. In Tools and Algorithms for Construction and Analysis of Systems (TACAS), pages 431-434, 1996.

6. V. Blondel, J. Hendrickx, A. Olshevsky, and J. Tsitsiklis. Convergence in multiagent coordination consensus and flocking. In Proceedings of the Joint fortyfourth IEEE Conference on Decision and Control and European Control Conference, pages 2996-3000, 2005.

7. K. M. Chandy, S. Mitra, and C. Pilotto. Formations of mobile agents with message loss and delay, 2007. preprint http://www.ist.caltech.edu/ mitras/research/ 2008/asynchcoord.pdf.

8. S. Chatterjee and E. Seneta. Towards consensus: some convergence theorems on repeated averaging. Journal of Applied Probability, 14(1):89-97, 1977.

9. S. Clavaski, M. Chaves, R. Day, P. Nag, A. Williams, and W. Zhang. Vehicle networks: achieving regular formation. In Proceedings of the American control Conference, 2003.

10. C. Dwork, N. Lynch, and L. Stockmeyer. Consensus in the presence of partial synchrony. Journal of the ACM, 35(2):288-323, 1988.

11. M. Hendriks. Model checking the time to reach agreement. In Formal Modeling and Analysis of Timed Systems, LNCS, pages 98-111. Springer, November 2005.

12. D. Kaynar, N. Lynch, S. Mitra, and S. Garland. TIOA Language. MIT Computer Science and Artificial Intelligence Laboratory, Cambridge, MA, 2005.

13. D. K. Kaynar, N. Lynch, R. Segala, and F. Vaandrager. The Theory of Timed I/O Automata. Synthesis Lectures on Computer Science. Morgan Claypool, November 2005. Also available as Technical Report MIT-LCS-TR-917.

14. L. Lamport. Real-time model checking is really simple. In Correct Hardware Design and Verification Methods, 13th IFIP WG (CHARME), volume 3725 of Lecture Notes in Computer Science, pages 162-175. Springer, 2005.

15. H. Lim, D. Kaynar, N. Lynch, and S. Mitra. Translating timed I/O automata specifications for theorem proving in PVS. In Proceedings of Formal Modelling and Analysis of Timed Systems (FORMATS'05), number 3829 in LNCS, Uppsala, Sweden, September 2005. Springer.

16. N. A. Lynch. Distributed Algorithms. Morgan Kaufmann Publishers Inc., 1996.

17. S. Mitra and K. M. Chandy. A formalized theory for verifying stability and convergence of automata in pvs. In In proceedings of Theorem Proving in Higher Order Logics (TPHOLS`08). LNCS, 2008. to appear.

18. R. Olfati-Saber, J. Fax, and R. Murray. Consensus and cooperation in networked multi-agent systems. Proceedings of the IEEE, 95(1):215-233, January 2007.

19. S. Owre, S. Rajan, J. Rushby, N. Shankar, and M. Srivas. PVS: Combining specification, proof checking, and model checking. In R. Alur and T. A. Henzinger, editors, Computer-Aided Verification, CAV'96, number 1102 in LNCS, pages 411414, New Brunswick, NJ, July/August 1996. Springer-Verlag.

20. J. N. Tsitsiklis. On the stability of asynchronous iterative processes. Theory of Computing Systems, 20(1):137-153, December 1987. 


\section{A Proofs for Lemmas in Sections 4 and 5}

Lemma 1. The collection $\left\{Q_{k}\right\}$ satisfies C1-3.

Proof. C1. We show that for any $k, l \in T$, if $l>k$ then $Q_{l} \subsetneq Q_{k}$. Fix $k, l \in T$ such that $l>k$. Consider any $\mathbf{s} \in Q_{l}$. By definition of $Q_{l}$, for all $s \in \mathcal{R}(\mathbf{s})$, $s \in P_{l}$. Applying $\mathrm{C} 1$, we obtain that for all $s \in \mathcal{R}(\mathbf{s}), s \in P_{k}$. By definition of $Q_{k}$, it follows that $\mathbf{s} \in Q_{k}$.

C2. From $\mathrm{C} 2$, exists $k$ such that $\forall s \in P_{k}, d\left(s, s^{*}\right)<\epsilon$. Hence, the result follows from the definition of $Q_{k}$.

C3. The result follows since $\mathbf{s}_{0}$.buffer is empty and $\mathbf{s}_{0} . y=\perp$ for all $\mathbf{s}_{0} \in Q_{0}$.

Lemma 5. $\forall m \in \mathbb{N}, \quad \mathcal{L}_{m, m i d} \cap \mathcal{R}_{m, m i d}=\mathcal{L}_{m+1,0} \cap \mathcal{R}_{m+1,0}$

Proof. Assume $s \in \mathcal{L}_{m, m i d} \cap \mathcal{R}_{m, m i d}$. By hypothesis $\forall i \in[N]$,

$$
e_{i}\left(s_{i}\right) \leq C \cdot \beta^{m}\left(1-\frac{1}{2^{m i d}}\right) \leq C \cdot \beta^{m+1} .
$$

Hence, the first condition of $\mathcal{L}_{m+1,0} \cap \mathcal{R}_{m+1,0}$ is satisfied by $s$. Noticing that agents 0 and $N$ never move, i.e. $e_{0}\left(s_{0}\right)=e_{N}\left(s_{N}\right)=0$, the second condition holds. Finally, by hypothesis the third condition holds. Hence, $s \in \mathcal{L}_{m+1,0} \cap \mathcal{R}_{m+1,0}$. The other direction follows from condition $\mathrm{C} 1$ of $\mathcal{L}, \mathcal{R}$ since $\mathcal{L}_{m+1,0} \subseteq \mathcal{L}_{m, \text { mid }}$ and $\mathcal{R}_{m+1,0} \subseteq \mathcal{R}_{m, m i d}$

Lemma 6. $\forall j<$ mid

(a) $\exists a_{1}$ such that $\forall s \stackrel{a_{1}}{\rightarrow} s^{\prime}$ and $\forall m \in \mathbb{N}, s \in \mathcal{L}_{m, j} \Rightarrow s^{\prime} \in \mathcal{L}_{m, j+1}$.

(b) $\exists a_{2}$ such that $\forall s \stackrel{a_{2}}{\rightarrow} s^{\prime}$ and $\forall m \in \mathbb{N}, s \in \mathcal{R}_{m, j} \Rightarrow s^{\prime} \in \mathcal{R}_{m, j+1}$.

Proof. Define $a_{1}=\left(j+1, \operatorname{avg}_{l, r}\right)$ with $(l, r) \in A_{j+1}$. Noticing that in the state $s^{\prime}$ the deviation error of $j+1$ is upper bounded by the convex combination of the deviation errors of $l, r$, the following chains of inequalities hold

$$
\begin{aligned}
e_{j+1}\left(s_{j+1}^{\prime}\right) & \leq \frac{r-(j+1)}{r-l} e_{l}\left(s_{l}\right)+\frac{(j+1)-l}{r-l} e_{r}\left(s_{r}\right) \\
& \leq \frac{r-(j+1)}{r-l}\left(1-\frac{1}{2^{l}}\right) C \cdot \beta^{m}+\frac{(j+1)-l}{r-l} C \cdot \beta^{m} \leq C \cdot \beta^{m}\left(1-\frac{1}{2^{j+1}}\right)
\end{aligned}
$$

where the last inequality follows from the concavity of the profile function $l p_{m, j+1}$. Hence, $s^{\prime} \in \mathcal{L}_{m, j+1}$. Similarly, we can prove $(b)$ by setting $a_{2}=(N-$ $\left.(j+1), \operatorname{avg}_{l, r}\right)$ with $(l, r) \in A_{N-(j+1)}$. 